\title{
Expression patterns and the prognostic value of the SMYD family members in human breast carcinoma using integrative bioinformatics analysis
}

\author{
JIANPING SONG $^{1}$, YANFENG LIU ${ }^{1}$, QIAN CHEN ${ }^{2}$, JINHUAN YANG ${ }^{1}$, \\ ZHENGCHEN JIANG ${ }^{1}$, HAO ZHANG ${ }^{1}$, ZHAOJIAN LIU ${ }^{3}$ and BIN JIN ${ }^{1}$ \\ Departments of ${ }^{1}$ General Surgery and ${ }^{2}$ Oncology, Qilu Hospital of Shandong University, Jinan, Shandong 250112; \\ ${ }^{3}$ Institute of Cell Biology, Shandong University School of Basic Medicine, Jinan, Shandong 250012, P.R. China
}

Received May 16, 2018; Accepted January 21, 2019

DOI: $10.3892 / \mathrm{ol} .2019 .10054$

\begin{abstract}
Suppressor of variegation, Enhancer of Zeste, Trithorax and Myeloid-Nervy-DEAF1 domain-containing (SMYD) proteins are a set of lysine methyltransferases involved in a range of diverse biological functions, including gene expression, and regulation of skeletal and cardiac-muscle development. These proteins may additionally serve roles in a number of different types of cancer. However, the roles of the five SMYD proteins, SMYD 1/2/3/4/5, their expression patterns and prognostic value remain unclear. In the present study, the transcriptional expression levels of the five SMYD proteins were compared with the survival data of patients with breast carcinoma (BC) from the ONCOMINE dataset, Breast Cancer Gene-Expression Miner v4.0, Kaplan-Meier Plotter, The Cancer Genome Atlas and cBioPortal. An increase in the SMYD2/3/5 mRNA expression levels and a decrease in SMYD1/4 mRNA expression levels in BC tissues compared with normal tissues were identified. Increased SMYD3 mRNA and decreased SMYD5 mRNA expression levels were associated with decreased levels of histological differentiation, according to the Scarff-Bloom-Richardson grading system. Kaplan-Meier curves demonstrated that the increased SMYD1/4 and decreased SMYD2/3 mRNA expression levels were associated with good relapse-free survival (RFS) in patients with BC. Furthermore, SMYD2 mRNA expression levels were associated with the RFS of patients with BC with metastatic relapse, and SMYD4 may serve as a tumor suppressor in patients with BC, as patients with increased SMYD4 mRNA expression levels had significantly better
\end{abstract}

Correspondence to: Dr Bin Jin, Department of General Surgery, Qilu Hospital of Shandong University, 107 Wenhua Xi Road, Jinan, Shandong 250112, P.R. China

E-mail: jinbin_qilu@163.com

Key words: Suppressor of variegation, Enhancer of Zeste, Trithorax and Myeloid-Nervy-DEAF1 domain-containing protein, breast carcinoma, prognosis, bioinformatic analyses, transcription
RFS compared with decreased SMYD4 mRNA expression levels. The present data suggested that SMYD2 and SMYD3 may be potential biomarkers for diagnosis of BC. Additionally, SMYD2 and SMYD4 may be potential prognostic indicators of patients with $\mathrm{BC}$.

\section{Introduction}

Breast carcinoma (BC) is one of the most common types of malignancy and is the second leading cause of cancer associated-mortalities among women (1). As in other lower or middle-income countries, BC is common in China, and its incidence is increasing. The disease occurs at a younger median age for Chinese women compared with western Caucasian women (2), and without a nationwide screening program, patients may be unaware of the importance of regular examination, which may lead to difficulty in treating patients. BC has a diverse and complex range of biological subtypes, which possess various unique clinical, pathological and molecular features (3). Based on the expression status of estrogen receptor (ER), progesterone receptor (PR) and receptor tyrosine-protein kinase erbB-2 (HER2), the molecular classification may offer better prognostic prediction and therapeutic outcomes (4).

Members of the Suppressor of variegation, Enhancer of Zeste, Trithorax (SET) and Myeloid-Nervy-DEAF1 (MYND) domain-containing protein (SMYD) family represent a group of proteins that contain the conserved SET and MYND domains (5). In the last decade, the SMYD protein family has attracted increasing interest due to its essential role in the development of the heart and muscle, and its potential role in the development of cancer (6). There are five members of the SMYD family, SMYD1, SMYD2, SMYD3, SMYD4 and SMYD5, and they all exhibit various biological functions, including developmental regulation and cancer (7). SMYD3 has been identified to be overexpressed in $>15$ types of cancer, and its overexpression may promote the proliferation of cancer cells (8). SMYD2 and SMYD4 expression has additionally been associated with cancer progression. SMYD2 was overexpressed in patients with leukemia with a poor prognosis (9), whereas, SMYD4 served as a tumor suppressor in BC (10). 
Advances in microarray technology have advanced DNA and RNA research, and has become an indispensable part of genomics (11). In the present study, thousands of gene expression or copy number analyses published online were collectively analyzed to investigate the expression of the SMYD family members in these databases to determine their clinical value in $\mathrm{BC}$.

\section{Materials and methods}

ONCOMINE data-mining analysis. ONCOMINE (www. oncomine.org) is an online cancer database, which was used to analyze the transcriptional alteration in different types of cancer (12). A comparison of the expression levels of the SMYD family members was made between clinical cancer samples and normal controls.

Breast Cancer Gene-Expression Miner v4.1 (bcGenExMiner v4.1). bcGenExMiner v4.1 (bcgenex.centregauducheau. $\mathrm{fr} / \mathrm{BC}$-GEM) is an online analysis tool, which contains 36 genomic datasets, with accompanying notes, (updated in December 2017) and was used to analyze the association between the mRNA expression levels of SMYDs and clinical parameters, including ER, PR and HER2 $(13,14)$.

Kaplan-Meier Plotter. Kaplan-Meier curves were created using the Kaplan-Meier Plotter (www.kmplot.com) to analyze the prognostic value of SMYD expression. Information on 3,951 clinical patients with $\mathrm{BC}$ were obtained from the website (15). Patients with $\mathrm{BC}$ were divided into two groups (high and low) based on the median expression level of SYMDs to evaluate the relapse-free survival (RFS) rate, according to the Kaplan-Meier curve. The JetSet best probe (16) set was selected if the target gene had more than one probe set.

The Cancer Genome Atlas data (TCGA) and cBioPortal. TCGA (cancergenome.nih.gov/) is a database, which collects the sequencing and pathological data of samples from 30 different types of cancer (17). cBioPortal (www.cbioportal. org) is a multi-functional visualization and analysis toolset, which may be used to anaylze gene expression of the cancer samples. The gene expression and pathological information of 1,108 cases were used from the BC dataset in cBioPortal for further analysis of expression of the SMYD family members $(18,19)$.

Statistical analysis. For the Elston-Ellis modification of the Scarff-Bloom-Richardson (SBR) grading system (20), significance was determined using a Welch's test with a post-hoc Dunnett-Tukey-Kramer's tests for pairwise comparison. The prognostic value of the SMYDs was calculated using a univariate Cox analysis (21) to generate the Forest plots. For the ONCOMINE datasets, a Student's t-test (22) was used to analyze the datasets. $\mathrm{P}<0.05$ or a 1.5 fold-change was considered to indicate a statistically significant difference. Welch's test, Dunnett-Tukey-Kramer's test and univariate Cox analysis were performed using bcGenExMiner v4.1, and Student's t-test was performed using ONCOMINE.

\section{Results}

Transcriptional expression levels of SMYDs in patients with $B C$. Using the datasets acquired from ONCOMINE, the mRNA expression levels of the SMYD family members in the BC samples were compared with the control samples (Fig. 1). The analysis demonstrated that SMYD3 was significantly upregulated in patients with BC in 27 databases. SMYD1 and SMYD4 were significantly downregulated in 24 and 10 of the datasets, respectively. In one dataset, Curtis et al (23), SMYD1 was identified to be downregulated in a number of different types of BC (medullary BC with a fold change of -2.12; ductal $\mathrm{BC}$ in situ with a fold change of -1.854 ; invasive $\mathrm{BC}$ with a fold change of -1.962; invasive lobular BC with a fold change of -1.903; and invasive ductal and invasive lobular BC with a fold change of -1.891 ; with a total fold change of -2.075 in all types of BC collectively) compared with expression in the normal tissue samples (Table I). By contrast, SMYD3 was upregulated in a number of types of $\mathrm{BC}$ (medullary $\mathrm{BC}$ with a fold change of 2.006; invasive ductal $\mathrm{BC}$ with a fold change of 2.526; invasive $\mathrm{BC}$ with a fold change of 2.342; invasive lobular $\mathrm{BC}$ with a fold change of 2.522; invasive ductal and invasive lobular $\mathrm{BC}$ with a fold change of 2.748; with a total fold change of 2.344 across all types of BC collectively) (18). The upregulation of SMYD2 in invasive ductal and invasive lobular BC (fold change of 1.449) and invasive ductal BC (fold change of 1.339) and the downregulation of SMYD4 in invasive BC (fold change of -1.807), invasive ductal and lobular BC (fold change of -1.889), and invasive ductal BC (fold change of -1.737) were identified in the TCGA dataset (Table I). No significant differences were identified for SMYD5 expression between the BC tissues and comparative normal tissues in either of the datasets (data not shown).

mRNA expression levels of the SMYD family members are significantly associated with various clinicopathological parameters of patients with $B C$. The mRNA expression levels of SMYDs between groups of patients according to different clinical parameters were compared using bcGenExMiner and the results are presented in Table II. SMYD3 was determined to be upregulated in patients with $\mathrm{BC}$ over the age of 51 Patients with BC with a positive nodal status presented increased SMYD5 mRNA expression levels compared with patients who had a negative nodal status.

SMYD2/3/5 were identified to be associated with ER and PR status. SMYD2/5 exhibited decreased expression levels in patients with ER and PR positive BC, whereas, SMYD3 demonstrated increased expression levels in patients with ER and PR positive BC. The transcriptional expression level of SMYD4 was decreased and the expression levels of SMYD5 were increased in patients with HER2 positive BC. Triple-negative $\mathrm{BC}$ (TNBC) is an aggressive form of BC where ER, PR and HER 2 expression is absent. The mRNA expression levels of SMYD2/5 in TNBC were increased, whereas, the expression of SMYD3 was decreased in patients with TNBC.

According to the SBR grading status criterion, it was demonstrated that the expression of all the SMYDs, with the exception of SMYD1, was associated with the SBR grade (Fig. 2). Decreased SMYD3 expression and increased SMYD5 expression suggested advanced SBR grade, whereas, SMYD2/4 


\begin{tabular}{|c|c|c|c|c|c|c|c|c|c|c|}
\hline \multirow{3}{*}{$\begin{array}{l}\text { Analysis type by cancer } \\
\text { Bladder Cancer }\end{array}$} & \multirow{2}{*}{\multicolumn{2}{|c|}{$\begin{array}{l}\text { Cancer } \\
\text { vs. } \\
\text { Normal } \\
\text { SMYD1 }\end{array}$}} & \multirow{2}{*}{\multicolumn{2}{|c|}{$\begin{array}{l}\text { Cancer } \\
\text { vs. } \\
\text { Normal } \\
\text { SMYD2 }\end{array}$}} & \multirow{2}{*}{\multicolumn{2}{|c|}{$\begin{array}{l}\text { Cancer } \\
\text { vs. } \\
\text { Normal } \\
\text { SMYD3 }\end{array}$}} & \multirow{2}{*}{\multicolumn{2}{|c|}{$\begin{array}{l}\text { Cancer } \\
\text { vs. } \\
\text { Normal } \\
\text { SMYD4 }\end{array}$}} & \multirow{2}{*}{\multicolumn{2}{|c|}{$\begin{array}{l}\text { Cancer } \\
\text { vs. } \\
\text { Normal } \\
\text { SMYD5 }\end{array}$}} \\
\hline & & & & & & & & & & \\
\hline & & & 4 & & 2 & & & & 2 & \\
\hline Brain and CNS Cancer & & 8 & 1 & 9 & 1 & 1 & & & 4 & 5 \\
\hline Breast Cancer & 2 & 24 & 2 & 2 & 27 & 1 & 1 & 10 & & 1 \\
\hline Cervical Cancer & & 1 & 4 & & 2 & & & & 1 & 1 \\
\hline Colorectal Cancer & & 5 & 16 & 1 & 18 & & 5 & 3 & 15 & \\
\hline Esophageal Cancer & & & 1 & 4 & 4 & & & & & 1 \\
\hline Gastric Cancer & & 1 & 3 & 1 & 2 & & 2 & & 4 & \\
\hline Head and Neck Cancer & & & 7 & & 8 & & & & 1 & \\
\hline Kidney Cancer & 1 & & 12 & 1 & 4 & 2 & 3 & & 1 & \\
\hline Leukemia & & & 7 & 7 & 5 & & 4 & & 1 & 1 \\
\hline Liver Cancer & & & 6 & & 3 & & & & & \\
\hline Lung Cancer & & & 5 & 1 & 3 & & & & 2 & 3 \\
\hline Lymphoma & & 2 & 6 & 8 & 5 & & 1 & & 1 & \\
\hline Melanoma & & & 2 & & 3 & & & & 2 & \\
\hline Myeloma & & & & & 1 & & & & 1 & \\
\hline Other Cancer & 1 & & 2 & 11 & 5 & & 1 & & 2 & \\
\hline Ovarian Cancer & & & 1 & 1 & 2 & & & & & \\
\hline Pancreatic Cancer & & & 2 & & 5 & & & & 1 & 1 \\
\hline Prostate Cancer & 1 & & 3 & & 1 & & & 2 & & \\
\hline Sarcoma & & & 2 & 4 & 7 & & 1 & & 3 & 1 \\
\hline Significant Unique Analyses & 5 & 41 & 86 & 47 & 108 & 4 & 18 & 15 & 40 & 14 \\
\hline Total Unique Analyses & & & & & 34 & & & & & \\
\hline
\end{tabular}

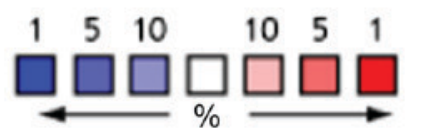

Figure 1. Expression levels of the SMYD family in different cancer types. Upregulation (red) and downregulation (blue) of the different members of the SMYD family of proteins in various different cancer types. All identified alterations in expression were considered statistically significant. $\mathrm{P}<0.05$. Cell color indicates the gene rank percentile. Image generated using ONCOMINE. SMYD, Suppressor of variegation, Enhancer of Zeste, Trithorax and Myeloid-Nervy-DEAF1 domain-containing; CNS, central nervous system.

exhibited a global significant difference with $\mathrm{P}<0.05$. No statistical significance of these SMYDs was observed using the Dunnett-Tukey-Kramer's test of each group in association with the SBR grade status (SBR1 vs. SBR2, SBR1 vs. SBR3 and SBR2 vs. SBR3; Table III).

Increased SMYD1/4 and decreased SMYD2/5 mRNA expression levels are associated with good RFS of patients with $B C$. The RFS of patients with BC was associated with the mRNA expression levels of SMYD1/2/4/5, which was identified by the Kaplan-Meier curve and a log-rank test $(\mathrm{P}<0.05)$ and stated below the plot is the number of patients that were at risk (Fig. 3). The patients with BC with increased SMYD1/4 mRNA expression levels or decreased SMYD2/5 mRNA expression levels were predicted to indicate good RFS.

Prognostic analysis of bcGenExMiner demonstrated the relevance between the mRNA expression levels of SMYD2 and the metastatic RFS (MRFS) in patients with BC was further analyzed by dataset (Table IV). An increased risk of metastatic relapse [hazard ratio $(\mathrm{HR})=1.25$; $95 \%$ confidence 
Table I. Different types of BC are associated with different alterations of SMYD expression (ONCOMINE database).

\begin{tabular}{|c|c|c|c|c|c|}
\hline \multicolumn{6}{|l|}{ A, SMYD1 } \\
\hline Author, year & Type of BC vs. breast & Fold change & P-value & t-test & (Refs.) \\
\hline \multirow[t]{6}{*}{ Curtis et al, 2012} & Medullary BC & -2.12 & $5.60 \times 10^{-24}$ & -11.703 & \multirow[t]{6}{*}{$(23)$} \\
\hline & Ductal BC in situ & -1.854 & 0.00000152 & -6.339 & \\
\hline & Invasive BC & -1.962 & $7.34 \times 10^{-8}$ & -6.389 & \\
\hline & Invasive lobular BC & -1.903 & $1.96 \times 10^{-19}$ & -9.805 & \\
\hline & Invasive ductal and invasive lobular BC & -1.891 & $2.34 \times 10^{-16}$ & -8.743 & \\
\hline & Total BC & -2.075 & $4.43 \times 10^{-08}$ & -7.198 & \\
\hline
\end{tabular}

B, SMYD2

\begin{tabular}{llccc}
\hline Author, year & \multicolumn{1}{c}{ Type of BC vs. breast } & Fold change & P-value & t-test \\
\hline $\begin{array}{l}\text { TCGA, accessed } \\
2018\end{array}$ & $\begin{array}{l}\text { Invasive ductal and invasive lobular } \\
\text { BC } \\
\text { Invasive lobular BC }\end{array}$ & 1.449 & 0.016 & 5.381 \\
& & 1.339 & $2.54 \times 10^{-23}$ & 14.652 \\
\hline
\end{tabular}

\section{C, SMYD3}

\begin{tabular}{llrrrr}
\hline Author, year & \multicolumn{1}{c}{ Type of BC vs. breast } & Fold change & P-value & t-test & (Refs.) \\
\hline \multirow{2}{*}{ Curtis et al, 2012 } & Medullary BC & 2.006 & $3.42 \times 10^{-8}$ & 6.841 & $(23)$ \\
& Invasive ductal BC & 2.526 & $2.05 \times 10^{-100}$ & 35.609 \\
& Invasive BC & 2.342 & 0.00000216 & 6.129 \\
& Invasive lobular BC & 2.522 & $2.41 \times 10^{-54}$ & 20.460 \\
& Invasive ductal and invasive lobular BC & 2.748 & $4.23 \times 10^{-35}$ & 17.426 \\
& Total BC & 2.344 & 0.00000443 & 6.808 \\
\hline
\end{tabular}

D, SMYD4

\begin{tabular}{|c|c|c|c|c|c|}
\hline Author, year & Type of BC vs. breast & Fold change & P-value & t-test & (Refs.) \\
\hline \multirow{3}{*}{$\begin{array}{l}\text { TCGA, accessed } \\
2018\end{array}$} & Invasive BC & -1.807 & $3.02 \times 10^{-23}$ & -12.039 & - \\
\hline & Invasive ductal and lobular BC & -1.889 & 0.0000791 & -10.652 & \\
\hline & Invasive ductal BC & -1.737 & $3.30 \times 10^{-24}$ & -14.280 & \\
\hline
\end{tabular}

BC, breast carcinoma; SMYD, Suppressor of variegation, Enhancer of Zeste, Trithorax and Myeloid-Nervy-DEAF1 domain-containing protein; TCGA, The Cancer Genome Atlas.

interval (CI) 1.17-1.34; $\mathrm{P}<0.0001]$ was observed in the patients with increased SMYD2 expression. Similarly, patients with increased SMYD2 expression had a decreased MRFS (HR $=1.38 ; 95 \%$ CI 1.21-1.56; P<0.0001; Fig. 4). The expression of SMYD1 (HR, 0.91; 95\% CI 0.77-1.09; $\mathrm{P}=0.3084)$, SMYD3 (HR, 0.94; 95\% CI 0.83-1.07; P=0.3514), SMYD4 (HR, 0.84; 95\% CI 0.69-1.02; $\mathrm{P}=0.0798$ ) and SMYD5 (HR, 1.10; 95\% CI $0.97-1.26 ; \mathrm{P}=0.1505)$ exhibited no association with MRFS of patients with $\mathrm{BC}$ (data not shown).

SMYD factors are significantly altered in patients with BC. A total of 365 samples out of 1,108 (37\%; data not shown) with invasive $\mathrm{BC}$ had altered expression levels of at least one of the SMYDs (3\% of samples with altered expression of SMYD1, $19 \%$ of samples with altered expression of SMYD2, 22\% of samples with altered expression of SMYD3, $7 \%$ of samples with altered expression of SMYD4 and 4\% of samples with altered expression of SMYD5; Fig. 5A). SMYD2/3 was increased in a portion of patients with BC. SMYD1/5 mRNA upregulation and SMYD4 mRNA downregulation were observed in certain patients with BC. SMYD members were rarely mutated in patients with $\mathrm{BC}$; however, the copy numbers of SMYD2/3 were positively associated with their mRNA expression levels (Fig. 5B and C). Patients with SMYD2/3 


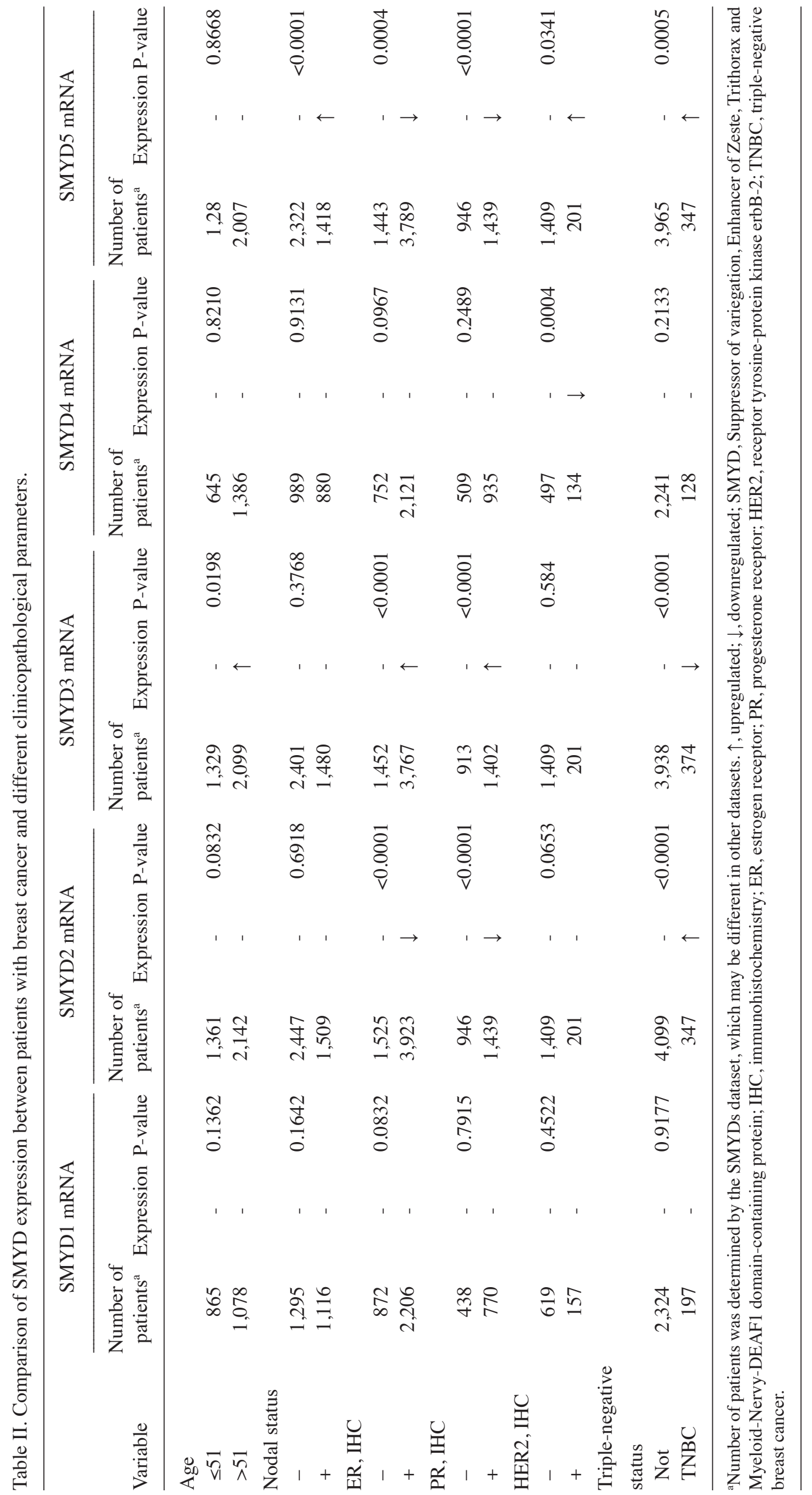




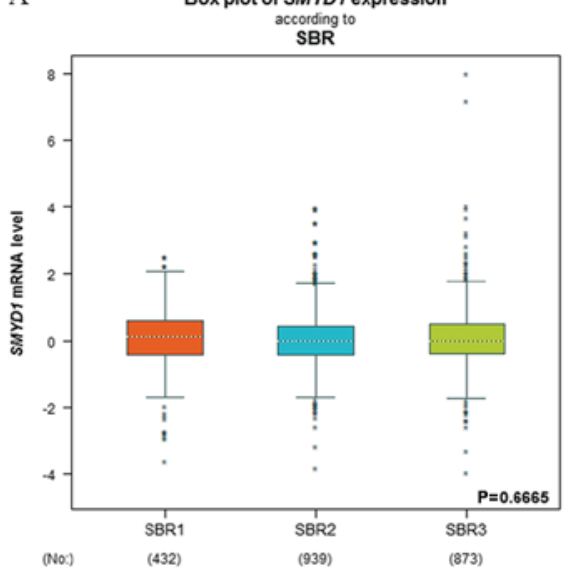

D

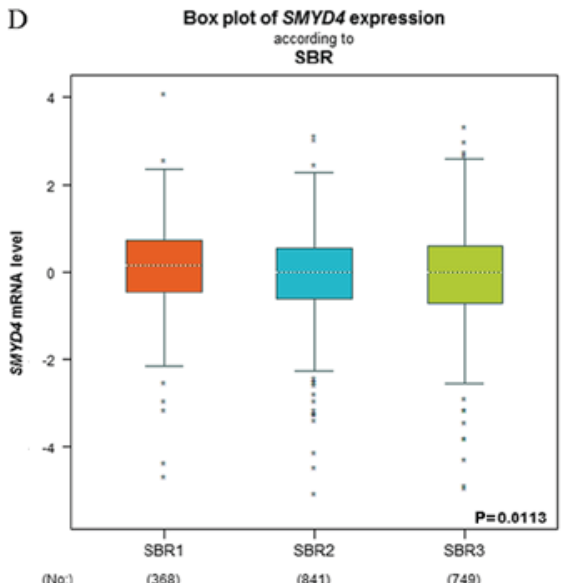

B

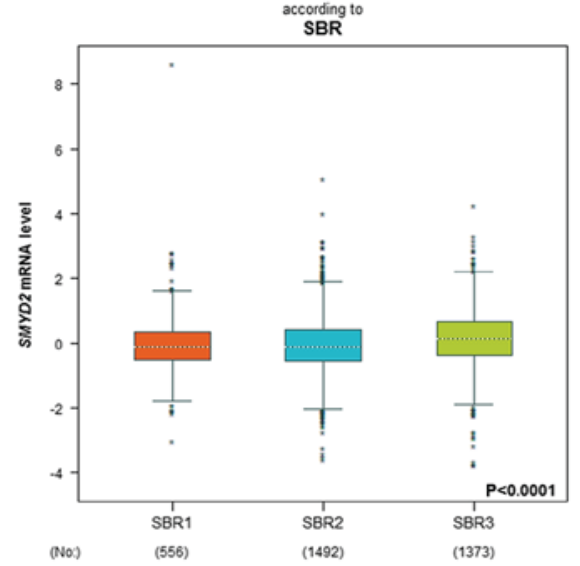

$\mathrm{E}$

E

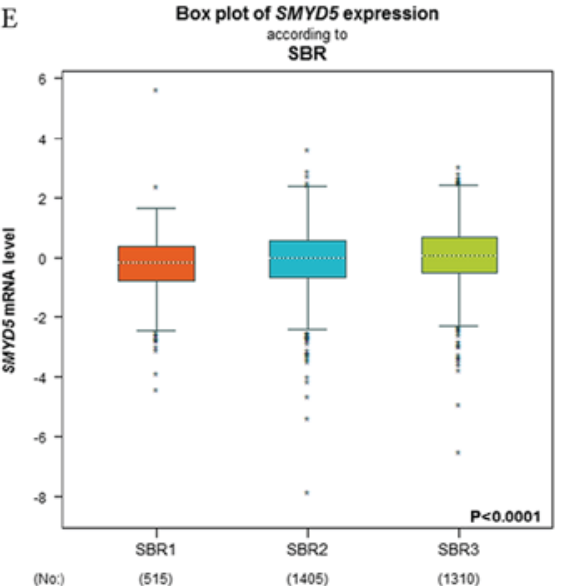

$\mathrm{C}$
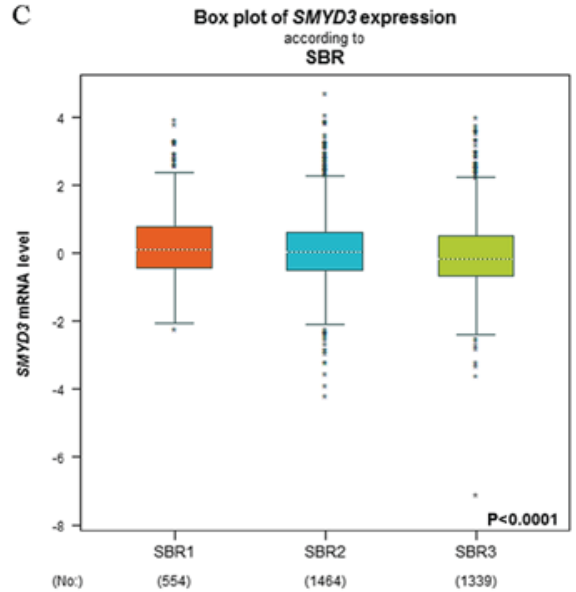

Figure 2. Association between mRNA expression levels of SMYDs and SBR grade status. Using Welch's test to generate P-values and Dunnett-Tukey-Kramer's tests for pairwise comparison, the difference between groups was assessed. (A) No association was determined between SMYD1 and SBR grade status of BC. (B) Expression of SMYD2 had a global significant difference in BC with a difference in SBR grade status. (C) Decreased SMYD3 expression levels are associated with advanced SBR grade status. (D) A global significant difference was determined for SMYD4 in BC with a difference in SBR grade status. (E) Increased SMYD5 expression levels are associated with an advanced SBR grade status. SMYD, Suppressor of variegation, Enhancer of Zeste, Trithorax and Myeloid-Nervy-DEAF1 domain-containing; SBR, Scarff-Bloom-Richardson; BC, breast carcinoma.

amplification had increased expression levels of SMYD2/3 compared with patients where increased SMYD2/3 amplification was not observed. However, no significant association was identified between the copy-number alternations of SMYD2/3 and the overall survival of patients with $\mathrm{BC}$ (SMYD2, $\mathrm{P}=0.958$; SMYD3, P=0.874; Fig. 5D and E).

\section{Discussion}

The SMYD proteins are abundantly expressed in cardiac and skeletal muscle, and were initially hypothesized to serve as epigenetic regulators for myogenesis and cardiomyocyte differentiation (8). SMYD proteins have attracted increasing interest in research following the identification of a potential role in the development of cancer, suggesting that the SMYD proteins may be potential therapeutic targets (24). To the best of the authors' knowledge, the present study is the first to analyze the mRNA expression levels and prognostic value of the SMYD family members in BC.

SMYD1, the first member of the SMYD family, has traditionally been known to function in skeletal muscle and myocardium development during the embryonic period (5).
Targeted SMYD1 deletion in mice disrupts the maturation of cardiomyocytes and formation of the right ventricle (25). Heparin binding growth factors (HDGF) possess mitogenic and angiogenic activities, and its expression is increased in the developing heart, tumor cell lines and certain normal tissues (26). HDGF has been demonstrated to repress the expression of SMYD1 in G-7 myoblast cells (27). HDGF is overexpressed in a number of types of human cancer, including hepatocellular carcinoma (28) and BC (29). The interaction of SMYD1 and HDGF suggests that SMYD1 may function in cancer development. In the present study, the mRNA expression levels of SMYD1 were decreased in patients with BC compared with normal breast tissue. Patients with BC with increased SMYD1 expression levels exhibited good RFS.

Numerous previous studies have demonstrated the involvement of SMYD2 in various cancer types. For example, SMYD2 has been demonstrated to promote TNBC progression (30). In the present study, patients with $\mathrm{BC}$ who exhibited decreased SMYD2 expression levels demonstrated improved RFS. SMYD2 represses the activity of cellular tumor antigen $\mathrm{p} 53$ by SMYD2-mediated methylation (31). SMYD2 may additionally downregulate the phosphatase and tensin (PTEN) pathway 
Table III. Dunnett-Tukey-Kramer's test for pairwise comparison in SBR criterion. SBR1, SBR2 and SBR3 means the SBR grade value.

\begin{tabular}{|c|c|c|c|}
\hline mRNA & Pairwise comparison of SBR & mRNA expression ${ }^{\mathrm{a}}$ & P-value \\
\hline \multirow[t]{3}{*}{ SMYD2 } & SBR1 vs. SBR2 & SBR $1=$ SBR 2 & $>0.10$ \\
\hline & SBR1 vs. SBR3 & SBR1<SBR3 & $<0.0001$ \\
\hline & SBR2 vs. SBR3 & SBR2<SBR3 & $<0.0001$ \\
\hline \multirow[t]{3}{*}{ SMYD3 } & SBR1 vs. SBR2 & SBR1>SBR2 & $<0.05$ \\
\hline & SBR1 vs. SBR3 & SBR1>SBR3 & $<0.0001$ \\
\hline & SBR2 vs. SBR3 & SBR2>SBR3 & $<0.001$ \\
\hline \multirow[t]{3}{*}{ SMYD4 } & SBR1 vs. SBR2 & SBR1>SBR2 & $<0.05$ \\
\hline & SBR1 vs. SBR3 & SBR1>SBR3 & $<0.05$ \\
\hline & SBR2 vs. SBR3 & $\mathrm{SBR} 2=\mathrm{SBR} 3$ & $>0.10$ \\
\hline \multirow[t]{3}{*}{ SMYD5 } & SBR1 vs. SBR2 & $\mathrm{SBR} 1<\mathrm{SBR} 2$ & $<0.05$ \\
\hline & SBR1 vs. SBR3 & SBR1<SBR3 & $<0.0001$ \\
\hline & SBR2 vs. SBR3 & SBR2<SBR3 & $<0.001$ \\
\hline
\end{tabular}

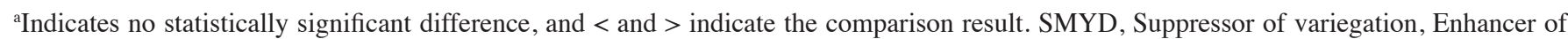
Zeste, Trithorax and Myeloid-Nervy-DEAF1 domain-containing protein; SBR, Scarff-Bloom-Richardson.
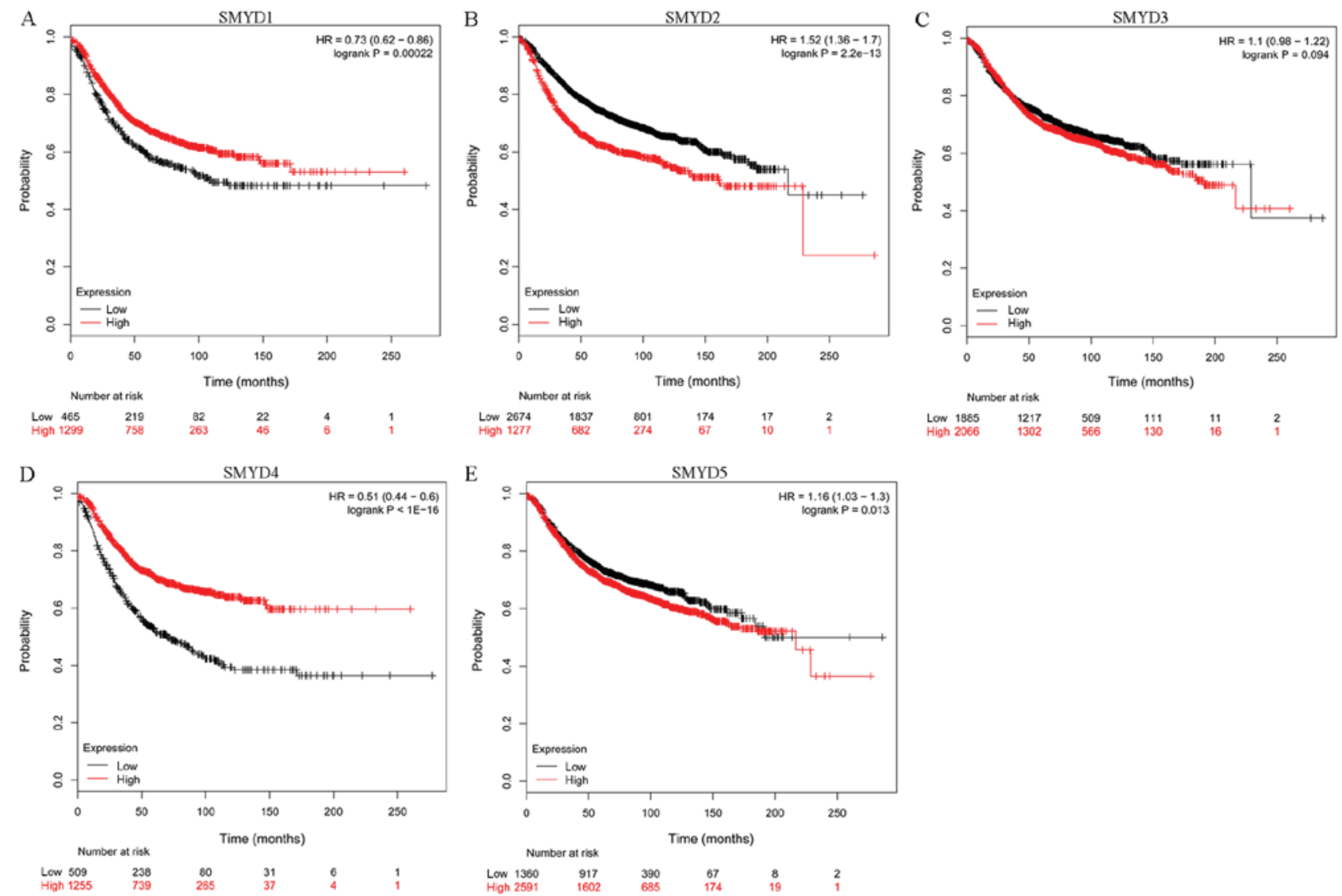

Figure 3. Association between the mRNA expression levels of the SMYD family members and the prognostic value in relapse free survival of patients with BC. (A) SMYD1 (228406_at; HR, 0.73; 95\% CI, 0.62-0.86; P=0.00022). (B) SMYD2 (212922_s_at; HR, 1.52; 95\%CI, 1.36-1.7; P=2.2x10-13). (C) SMYD3 (218788_s_at; HR, 1.1; 95\% CI, 0.98-1.22; P=0.094). (D) SMYD4 (229175_at; HR, 0.51; 95\% CI, 0.44-0.6; P=1x10-16). (E) SMYD5 (209516_at; HR, 1.16; 95\% CI, 1.03-1.3; $\mathrm{P}=0.013)$. SMYD, Suppressor of variegation, Enhancer of Zeste, Trithorax and Myeloid-Nervy-DEAF1 domain-containing; HR, hazard ratio; CI, confidence interval.

through the methylation of PTEN (32). These previous studies examined the important role of SMYD2 in cancer. In the present study, SMYD2 was significantly increased in patients with BC and those with metastatic relapse. Decreased SMYD2 
mRNA expression levels were associated with good RFS and, thus, raises the possibility that SMYD2 serves as a diagnostic biomarker in $\mathrm{BC}$ diagnosis and as a prognostic indicator for patients with $\mathrm{BC}$ with metastatic relapse.

SMYD3 is considered an oncogene in a number of different types of cancer. SMYD3 is regulated by $\beta$-catenin/transcription factor- 4 transcription apparatus in gastric cancer (33). SMYD3 mediated methylation of mitogen-activated protein kinase (MAPK) kinase kinase 2, which stimulated MAPK signaling and promoted the formation of Ras-driven carcinomas (34). SMYD3 may additionally influence distinct oncogenic processes by serving as a gene-specific transcriptional regulator (35). SMYD3 has been demonstrated to promote the development of $\mathrm{BC}(36,37)$ and is associated with familial $\mathrm{BC}(38)$. In the present study, it was identified that SMYD3 was amplified in patients with $\mathrm{BC}$; however, it was not associated with the RFS of patients with BC. Additionally, it was observed that advanced SBR grade was associated with decreased SMYD3 mRNA expression levels. Therefore, SMYD3 may be used as a biomarker for diagnosis of $\mathrm{BC}$ and as an indicator of the SBR grade of BC tissue.

Less is known regarding the role of SMYD4 in cancer development. Hu et al (10) demonstrated that SMYD4 may serve as a tumor suppressor gene in BC. However, to the best of the authors' knowledge, there is no information regarding an association between SMYD4 and the prognosis of patients with BC. In the present study, increased SMYD4 mRNA expression levels were associated with good RFS in patients with BC. As a result, it is possible that SMYD4 may serve as a good prognostic indicator.

Similar to SMYD4, there are comparatively fewer studies investigating the role of SMYD5 in cancer development. At present, previous studies on SMYD5 focused on its role in embryonic stem (ES) cells. SMYD5 primarily serves a role in the differentiation of ES cells $(39,40)$. However, the depletion of SMYD5 in human colon and lung cancer cells resulted in increased tumor growth and the upregulation of genes associated with colon and lung cancer (39). In the present study, it was demonstrated that the SMYD5 mRNA expression levels were decreased in patients with ER/PR-positive $\mathrm{BC}$ compared with those with ER/PR-negative BC and increased in patients with HER2-positive BC and TNBC. Increased SMYD5 mRNA expression levels were associated with advanced SBR grade. Therefore, SMYD5 may serve as a potential oncogene in $\mathrm{BC}$.

In conclusion, the SMYD family may function in the development of BC. Previous studies investigating the functions of the SMYD family members in cancer are rare and the mechanisms regarding the differential expression pattern of its family members in BC remain unclear. In the present study, the expression of SMYDs was systemically analyzed to evaluate their clinical and prognostic value in BC. The present findings suggested that SMYD2/3 may serve as potential diagnostic biomarkers and therapeutic targets for BC. In addition, the present data suggested that SMYD4 may serve as a potential prognostic marker of survival in patients with $\mathrm{BC}$. The increases in SMYD1/5 mRNA expression levels observed in the present data suggested a potential role for SMYD1/5 in the development of BC. 

A SMYD2: Forest plot

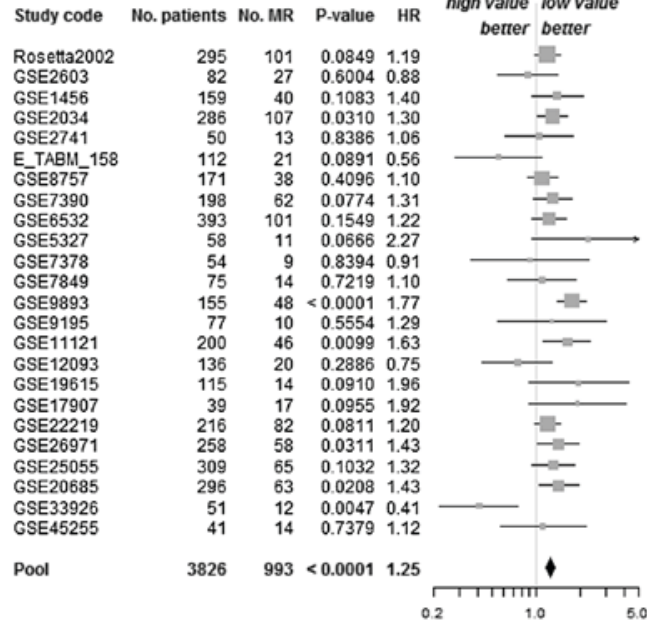

B $\quad S M Y D 2$

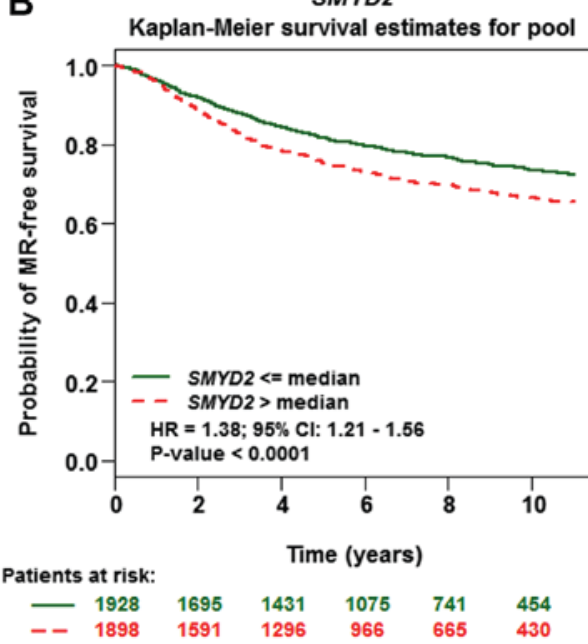

Figure 4. SMYD2 mRNA expression levels are negatively associated with MR-free survival in patients with breast carcinoma. (A) Univariate Cox analysis of the expression of SMYD2 and MR is presented in a forest plot. (B) Kaplan-Meier curve depicting a negative association between the mRNA expression level of SMYD2 and MR-free survival. SMYD, Suppressor of variegation, Enhancer of Zeste, Trithorax and Myeloid-Nervy-DEAF1 domain-containing; HR, hazard ratio; $\mathrm{CI}$, confidence interval; MR, metastatic relapse.

A

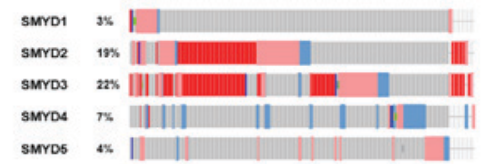

- Inframe mutation (unknown significance) " Missense mutation (unknown significance) " "Truncating mutation (unkenown significance)

| Amplification | Deep deletion I mRNA upregulation |] mRNA downregulation || No alterations - Not sequenced
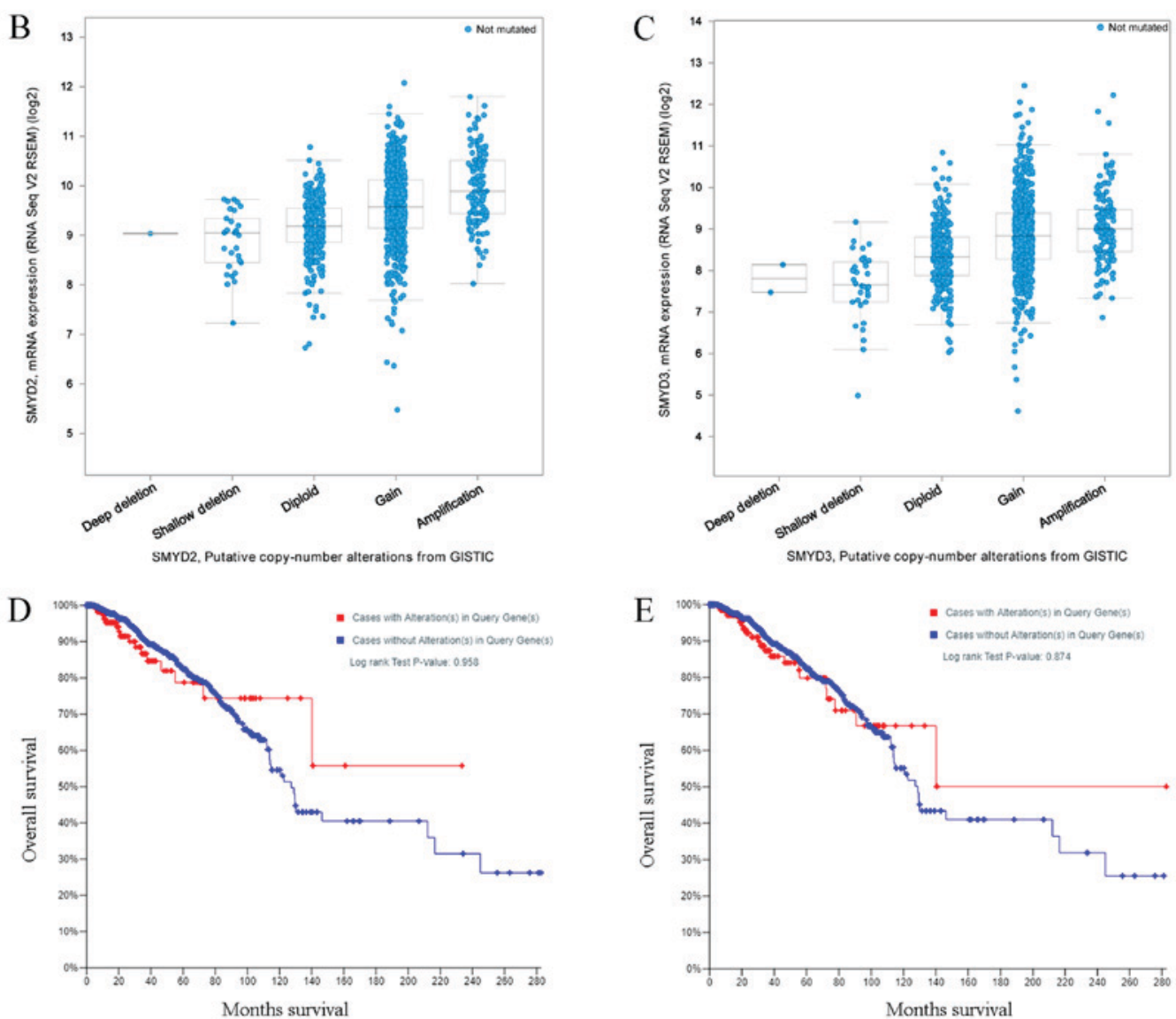

Figure 5. Analysis of the expression and mutation of the SMYD family members in invasive breast carcinoma (cBioPortal). (A) Various genetic alterations in samples of the SMYD family members. Association between the copy number of (B) SMYD2 and (C) SMYD3 and their respective mRNA expression levels. (D) Overall survival rate with and without (D) SMYD2 and (E) SMYD3 copy-number alternations. SMYD, Suppressor of variegation, Enhancer of Zeste, Trithorax and Myeloid-Nervy-DEAF1 domain-containing. 


\section{Acknowledgements}

The authors would like to acknowledge Mr Yanfeng Liu (Qilu Hospital of Shandong University, Jinan, China) and Professor Zhaojian Liu (Institute of Cell Biology, Shandong University School of Basic Medicine, Jinan, China) for editing the manuscript and assisting with data analyses.

\section{Funding}

The present study was supported by The National Natural Science Foundation of China (grant no. 81571367), The Shandong Scientific and Technological Research Program (grant nos. 2016GSF201082, 2017GSF218021 and 2018GSF118191; China) and Shandong Natural Science Foundation (grant no. ZR2014HQ043; China).

\section{Availability of data and materials}

The datasets used and analyzed during the present study are available from the corresponding author on reasonable request.

\section{Authors' contributions}

JS, BJ and ZL designed and conceived the study. YL wrote the manuscript. QC, JY, ZJ and HZ reviewed the manuscript for important intellectual content YL, QC and JY assisted with acquisition of data. ZJ and $\mathrm{HZ}$ were involved in the analysis and interpretation of data. All authors read and approved the final manuscript.

\section{Ethics approval and consent to participate}

Not applicable.

\section{Patient consent for publication}

Not applicable.

\section{Competing interests}

The authors declare that they have no competing interests.

\section{References}

1. DeSantis C, Siegel R, Bandi P and Jemal A: Breast cancer statistics, 2011. CA Cancer J Clin 61: 409-418, 2011.

2. Fan L, Strasser-Weippl K, Li JJ, St LJ, Finkelstein DM, Yu KD, Chen WQ, Shao ZM and Goss PE: Breast cancer in China. Lancet Oncol 15: e279-e289, 2014.

3. Siegel RL, Miller KD and Jemal A: Cancer statistics, 2015. CA Cancer J Clin 65: 5-29, 2015

4. Onitilo AA, Engel JM, Greenlee RT and Mukesh BN: Breast cancer subtypes based on ER/PR and Her2 expression: Comparison of clinicopathologic features and survival. Clin Med Res 7: 4-13, 2009.

5. Du SJ, Tan X and Zhang J: SMYD proteins: Key regulators in skeletal and cardiac muscle development and function. Anat Rec (Hoboken) 297: 1650-1662, 2014.

6. Doughan M, Spellmon N, Li C and Yang Z: SMYD proteins in immunity: Dawning of a new era. AIMS Biophys 3: 450-455, 2016.

7. Leinhart K and Brown M: SET/MYND lysine methyltransferases regulate gene transcription and protein activity. Genes (Basel) 2: $210-218,2011$
8. Spellmon N, Holcomb J, Trescott L, Sirinupong N and Yang Z: Structure and function of SET and MYND domain-containing proteins. Int J Mol Sci 16: 1406-1428, 2015.

9. Sakamoto LH, Andrade RV, Felipe MS, Motoyama AB and Pittella SF: SMYD2 is highly expressed in pediatric acute lymphoblastic leukemia and constitutes a bad prognostic factor. Leuk Res 38: 496-502, 2014.

10. Hu L, Zhu YT, Qi C and Zhu YJ: Identification of Smyd4 as a potential tumor suppressor gene involved in breast cancer development. Cancer Res 69: 4067-4072, 2009.

11. Sealfon SC and Chu TT: RNA and DNA microarrays. Methods Mol Biol 671: 3-34, 2011.

12. Rhodes DR, Yu J, Shanker K, Deshpande N, Varambally R, Ghosh D, Barrette T, Pandey A and Chinnaiyan AM: ONCOMINE: A cancer microarray database and integrated data-mining platform. Neoplasia 6: 1-6, 2004.

13. Jézéquel $P$, Campone $M$, Gouraud $W$, Guérin-Charbonnel $C$, Leux C, Ricolleau G and Campion L: bc-GenExMiner: An easy-to-use online platform for gene prognostic analyses in breast cancer. Breast Cancer Res Treat 131: 765-775, 2012.

14. Jézéquel P, Frénel JS, Campion L, Guérin-Charbonnel C, Gouraud W, Ricolleau G and Campone M: bc-GenExMiner 3.0: New mining module computes breast cancer gene expression correlation analyses. Database (Oxford) 2013: bas060, 2013.

15. Györffy B, Lanczky A, Eklund AC, Denkert C, Budczies J, Li Q and Szallasi Z: An online survival analysis tool to rapidly assess the effect of 22,277 genes on breast cancer prognosis using microarray data of 1,809 patients. Breast Cancer Res Treat 123: 725-731, 2010.

16. Li Q, Birkbak NJ, Gyorffy B, Szallasi Z and Eklund AC: Jetset: Selecting the optimal microarray probe set to represent a gene. BMC Bioinformatics 12: 474, 2011.

17. Cancer Genome Atlas Network: Comprehensive molecular portraits of human breast tumours. Nature 490: 61-70, 2012.

18. Gao J, Aksoy BA, Dogrusoz U, Dresdner G, Gross B, Sumer SO, Sun Y, Jacobsen A, Sinha R, Larsson E, et al: Integrative analysis of complex cancer genomics and clinical profiles using the cBioPortal. Sci Signal 6: 2013.

19. Cerami E, Gao J, Dogrusoz U, Gross BE, Sumer SO, Aksoy BA, Jacobsen A, Byrne CJ, Heuer ML, Larsson E, et al: The cBio cancer genomics portal: An open platform for exploring multidimensional cancer genomics data. Cancer Discov 2: 401-404, 2012.

20. Martinazzi M, Zampatti C, Crivelli F, Zampieri A and Martinazzi S: Scarff-bloom-richardson histoprognostic grading correlates with the immunohistochemical expressions of genomic alterations in infiltrating ductal carcinomas (nos) of the breast. Oncol Rep 1: 1087-1091, 1994.

21. Nicolai P, Redaelli de Zinis LO, Tomenzoli D, Barezzani MG, Bertoni F, Bignardi M and Antonelli AR: Prognostic determinants in supraglottic carcinoma: Univariate and Cox regression analysis. Head Neck 19: 323-34, 1997.

22. Hsu CL and Lee WC: Detecting differentially expressed genes in heterogeneous diseases using half student's t-test. Int J Epidemiol 39: 1597-1604, 2010.

23. Curtis C, Shah SP, Chin SF, Turashvili G, Rueda OM, Dunning MJ, Speed D, Lynch AG, Samarajiwa S, Yuan Y, et al: The genomic and transcriptomic architecture of 2,000 breast tumours reveals novel subgroups. Nature 486: 346-352, 2012.

24. Gottlieb PD, Pierce SA, Sims RJ, Yamagishi H, Weihe EK, Harriss JV, Maika SD, Kuziel WA, King HL, Olson EN, et al: Bop encodes a muscle-restricted protein containing MYND and SET domains and is essential for cardiac differentiation and morphogenesis. Nat Genet 31: 25-32, 2002.

25. Everett AD: Identification, cloning, and developmental expression of hepatoma-derived growth factor in the developing rat heart. Dev Dyn 222: 450-458, 2001.

26. Al-Shar'i NA and Alnabulsi SM: Explaining the autoinhibition of the SMYD enzyme family: A theoretical study. J Mol Graph Model 68: 147-157, 2016.

27. Yang $J$ and Everett AD: Hepatoma-derived growth factor represses SET and MYND domain containing 1 gene expression through interaction with $\mathrm{C}$-terminal binding protein. J Mol Biol 386: 938-950, 2009.

28. Hu TH, Huang CC, Liu LF, Lin PR, Liu SY, Chang HW, Changchien CS, Lee CM, Chuang JH and Tai MH: Expression of hepatoma-derived growth factor in hepatocellular carcinoma. Cancer 98: 1444-1456, 2003. 
29. Chen SC, Kung ML, Hu TH, Chen HY, Wu JC, Kuo HM Tsai HE, Lin YW, Wen ZH, Liu JK, et al: Hepatoma-derived growth factor regulates breast cancer cell invasion by modulating epithelial-mesenchymal transition. J Pathol 228: 158-169, 2012.

30. Li LX, Zhou JX, Calvet JP, Godwin AK, Jensen RA and Li X: Lysine methyltransferase SMYD2 promotes triple negative breast cancer progression. Cell Death Dis 9: 326, 2018.

31. Huang J, Perez-Burgos L, Placek BJ, Sengupta R, Richter M, Dorsey JA, Kubicek S, Opravil S, Jenuwein T and Berger SL: Repression of p53 activity by Smyd2-mediated methylation. Nature 444: 629-632, 2006

32. Nakakido M, Deng Z, Suzuki T, Dohmae N, Nakamura $Y$ and Hamamoto R: Dysregulation of AKT pathway by SMYD2-mediated lysine methylation on PTEN. Neoplasia 17: 367-373, 2015

33. Wang T, Wu H, Liu S, Lei Z, Qin Z, Wen L, Liu K, Wang X, Guo Y, Liu Q, et al: SMYD3 controls a Wnt-responsive epigenetic switch for ASCL2 activation and cancer stem cell maintenance. Cancer Lett 430: 11-24, 2018

34. Mazur PK, Reynoird N, Khatri P, Jansen PW, Wilkinson AW, Liu S, Barbash O, Van Aller GS, Huddleston M, Dhanak D, et al: SMYD3 links lysine methylation of MAP3K2 to Ras-driven cancer. Nature 510: 283-287, 2014.

35. Kim JM, Kim K, Schmidt T, Punj V, Tucker H, Rice JC Ulmer TS and An W: Cooperation between SMYD3 and PC4 drives a distinct transcriptional program in cancer cells. Nucleic Acids Res 43: 8868-8883, 2015.
36. Tsai CH, Chen YJ, Yu CJ, Tzeng SR, Wu IC, Kuo WH, Lin MC, Chan NL, Wu KJ and Teng SC: SMYD3-Mediated H2A.Z.1 Methylation promotes cell cycle and cancer proliferation. Cancer Res 76: 6043-6053, 2016.

37. Hamamoto R, Silva FP, Tsuge M, Nishidate T, Katagiri T, Nakamura Y and Furukawa Y: Enhanced SMYD3 expression is essential for the growth of breast cancer cells. Cancer Sci 97: 113-118, 2006.

38. Frank B, Hemminki K, Wappenschmidt B, Klaes R, Meindl A, Schmutzler RK, Bugert P, Untch $M$, Bartram CR and Burwinkel B: Variable number of tandem repeats polymorphism in the SMYD3 promoter region and the risk of familial breast cancer. Int J Cancer 118: 2917-2918, 2006.

39. Kidder BL, He R, Wangsa D, Padilla-Nash HM, Bernardo MM, Sheng S, Ried T and Zhao K: SMYD5 Controls heterochromatin and chromosome integrity during embryonic stem cell differentiation. Cancer Res 77: 6729-6745, 2017.

40. Kidder BL, Hu G, Cui K and Zhao K: SMYD5 regulates H4K20me3-marked heterochromatin to safeguard ES cell self-renewal and prevent spurious differentiation. Epigenetics Chromatin 10: 8, 2017.

(i) (3) This work is licensed under a Creative Commons Attribution-NonCommercial-NoDerivatives 4.0 International (CC BY-NC-ND 4.0) License. 